\title{
Rickettsia japonica Infections in Humans, Zhejiang Province, China, 2015
}

\author{
Qunying Lu, Jianping Yu, Liqun Yu, \\ Yanjun Zhang, Yitao Chen, \\ Meiai Lin, Xiaofei Fang
}

We investigated 16 Japanese spotted fever cases that occurred in southeastern China during September-October 2015. Patients had fever, rash, eschar, and lymphadenopathy. We confirmed 9 diagnoses and obtained 2 isolates with high identity to Rickettsia japonica strain YH. R. japonica infection should be considered for febrile patients in China.

$R$ ickettsia japonica is a member of the spotted fever group rickettsiae (SFGR) that causes tickborne Japanese spotted fever (JSF). First reported in Japan's Tokushima Prefecture in $1984(1,2)$, JSF has been recognized in multiple countries of Asia, including Japan, South Korea, the Philippines, and Thailand (3-5). In China, 4 species of SFGR have been reported to cause human infection: $R$. heilongjiangensis, $R$. sibirica subspecies sibirica BJ-90, Candidatus $\mathrm{R}$. tarasevichiae, and $R$. raoultii (6). In this report, we investigated the causative agent of 16 JSF cases that occurred in southeastern China in late 2015.

\section{The Study}

The ethics committee of the Zhejiang Province Center for Disease Control and Prevention, Hangzhou, China, approved this research. During September-October 2015, a total of 16 febrile patients were hospitalized at Linan First People's Hospital (Linan, China). All these patients lived in the Xitianmu Mountain area of Linan in Zhejiang Province. Besides fever $\left(38.8^{\circ} \mathrm{C}-40.3^{\circ} \mathrm{C}\right)$, clinical signs of disease in these patients included rashes on the trunk and limbs (15/16) and an eschar (11/16) (Table). In those with eschar, lymphadenopathy was found at the site of the draining lymph node (6/11). Five patients had rash and no eschar. Laboratory results revealed that all patients' leukocyte levels were within reference ranges, but a high percentage of neutrophils (12/16 patients) and minor hepatic transaminase elevation (11/16 patients) were also observed. All 16 patients were treated with doxycycline

Author affiliations: Zhejiang Province Center for Disease Control and Prevention, Hangzhou, China (Q. Lu, Y. Zhang, X. Fang); Linan First People's Hospital, Linan, China (J. Yu, L. Yu); Zhejiang Chinese Medical University, Hangzhou (Y. Chen, M. Lin)

DOI: https://doi.org/10.3201/eid2411.170044 or azithromycin and were cured, and no patient experienced severe illness.

With patient consent, we collected acute-phase $(\mathrm{n}=$ $16)$ and convalescent-phase $(n=14)$ whole blood specimens and sent them to Zhejiang Province Center for Disease Control and Prevention for laboratory confirmation of Rickettsia infection. We extracted DNA from acutephase blood specimens by using the DNeasy Blood \& Tissue Kit (QIAGEN, Hilden, Germany). We used nested PCR to amplify the partial groEL genes of SFGR, typhus group rickettsiae, and Orientia tsutsugamushi bacteria (online Technical Appendix, https://wwwnc. cdc.gov/EID/article/24/11/17-0044-Techapp1.pdf). The targeted fragments $(217 \mathrm{bp}$ ) were present in the blood specimens from 9 patients (Table). We sequenced these fragments and analyzed them using BLAST (http://www. ncbi.nlm.nih.gov/BLAST), and all had $100 \%$ identity to $R$. japonica $\mathrm{YH}$ prototype strain (GenBank accession no. NC016050) $(7,8)$. All specimens were negative for typhus group rickettsiae and $O$. tsutsugamushi rickettsia DNA by PCR.

We also inoculated $200 \mu \mathrm{L}$ of acute-phase blood specimens onto HL60 and DH82 cells in 25-mL flasks and cultured at $37^{\circ} \mathrm{C}$. Cytopathic effect was not observed with inoculated HL60 cells, but inoculated DH82 cells exfoliated completely by 4 weeks of culture. We also performed indirect immunofluorescence assays (IFAs) every 2 days to access SFGR growth (online Technical Appendix). Two of the inoculated cultures exhibited bright fluorescent apple-green, rod-shaped particles (Table) after 3 weeks of culture, confirming SFGR infection for 2 patients. We then extracted DNA from the 2 SFGR-positive cultures (LA4/2015 and LA16/2015) and amplified and sequenced a 2,493-bp fragment containing the full-length sequences of SFGR groES and groEL (GenBank accession nos. KY073364-5) and a 609-bp fragment containing the partial rickettsial ompA gene sequence (GenBank accession nos. KY347792-3; online Technical Appendix Table). These sequences were found to be $100 \%$ identical to the corresponding sequences of $R$. japonica $\mathrm{YH}$.

We used IFAs with bacterial substrate antigens $R$. japonica (HL-60 cells infected with LA4/2015) and $R$. rickettsii (FOCUS Diagnostics Inc., Cyprus, CA, USA) to test patients for specific antibodies, and in all 16 patient serum samples, we detected SFGR IgG. All paired serum samples $(n=14)$ showed a $>4$-fold increase in titer against SFGR (Table). The 2 patients we did not receive 
Table. Clinical data of febrile patients with Japanese spotted fever diagnoses, Linan, China, 2015*

\begin{tabular}{|c|c|c|c|c|c|c|c|c|c|c|c|}
\hline \multirow{2}{*}{$\begin{array}{l}\text { Patient } \\
\text { no. }\end{array}$} & \multirow{2}{*}{$\begin{array}{l}\text { Age, } \\
\text { y/sex }\end{array}$} & \multirow[b]{2}{*}{ Tick bite } & \multirow[b]{2}{*}{ Rash } & \multirow[b]{2}{*}{ Eschar } & \multirow[b]{2}{*}{ Lymphadenopathy } & \multicolumn{2}{|c|}{ Days after onset } & \multirow{2}{*}{$\begin{array}{c}\text { PCR, } † \\
\text { AP }\end{array}$} & \multicolumn{2}{|c|}{ IFA titer } & \multirow[b]{2}{*}{ Isolation } \\
\hline & & & & & & $\mathrm{AP}$ & $\mathrm{CP}$ & & AP & $\mathrm{CP}$ & \\
\hline 1 & $53 / \mathrm{M}$ & Not known & Yes & No & No & 6 & 42 & - & $<64$ & 4,096 & - \\
\hline 2 & $62 / \mathrm{M}$ & Not known & Yes & Yes & No & 5 & 58 & + & $<64$ & 2,048 & - \\
\hline 3 & $52 / \mathrm{M}$ & Yes & Yes & Yes & Yes & 5 & 74 & + & 64 & 4,096 & - \\
\hline 4 & $79 / F$ & Not known & Yes & No & No & 4 & 60 & + & 128 & 9,192 & + \\
\hline 5 & $67 / F$ & No & Yes & No & No & 6 & 75 & + & 512 & 4,096 & - \\
\hline 6 & $62 / F$ & Yes & Yes & Yes & No & 7 & 70 & - & $<64$ & 1,024 & - \\
\hline 7 & $53 / F$ & Yes & No & Yes & No & 7 & 57 & - & 64 & 1,024 & - \\
\hline 8 & $63 / F$ & Yes & Yes & Yes & Yes & 10 & 67 & - & $<64$ & 4,096 & - \\
\hline 9 & $57 / \mathrm{M}$ & Yes & Yes & Yes & No & 2 & 45 & + & 64 & 9,192 & - \\
\hline 10 & $51 / F$ & Yes & Yes & Yes & Yes & 6 & $\S$ & + & 64 & $\S$ & - \\
\hline 11 & 53/M & Yes & Yes & Yes & No & 3 & $\S$ & + & $<64$ & $\S$ & - \\
\hline 12 & $45 / F$ & Yes & Yes & Yes & Yes & 5 & 70 & + & $<64$ & 4,096 & - \\
\hline 13 & $33 / F$ & Yes & Yes & Yes & Yes & 6 & 42 & - & 64 & 2,048 & - \\
\hline 14 & $30 / F$ & Not known & Yes & No & No & 4 & 46 & - & 128 & 2,048 & - \\
\hline 15 & $36 / F$ & Not known & Yes & No & No & 5 & 54 & - & $<64$ & 2,048 & - \\
\hline 16 & $67 / F$ & Yes & Yes & Yes & Yes & 0 & 60 & + & $<64$ & 9,192 & + \\
\hline
\end{tabular}

${ }^{*} \mathrm{AP}$, acute phase; CP, convalescent phase; IFA, indirect immunofluorescence assay; + , positive test result; - , negative test result.

†PCR amplifying 217-bp sequence of Rickettsia japonica groEL gene.

‡Detection of IgG against spotted fever group rickettsiae.

$\S$ Convalescent-phase serum samples not obtained.

convalescent-phase serum specimens from were positive for $R$. japonica by PCR.

All serum specimens were negative for $O$. tsutsugamushi IgG. Some convalescent-phase serum specimens had low-titer reactions to $R$. typhi bacterial antigen.

\section{Conclusions}

The 4 SFGR species $R$. japonica, $R$. heilongjiangensis, $R$. rhipicephali, and $R$. massiliae have been identified in Haemaphysalis longicornis and Rhipicephalus haemaphysaloides ticks in Zhejiang Province (9-11), indicating a potential for these species to infect humans in China. In our research, we determined the etiologic agent of $16 \mathrm{JSF}$ cases and isolated $2 R$. japonica rickettsiae. The prototype strain $R$. japonica YH was isolated in Japan in 1985 (1). After $\approx 30$ years, only a few $R$. japonica isolates have been isolated from patients in China: 2 from our research and 1 from $\mathrm{Li}$ et al. (12). Our findings indicate that the fulllength groES and groEL genes and the partial ompA gene sequences were $100 \%$ identical to $R$. japonica $\mathrm{YH}$, suggesting that the $R$. japonica genome has been relatively conserved. Nine patients had clinically confirmed JSF, displaying fever, rash, eschar, and lymphadenopathy; these signs and symptoms were similar to those seen in JSF patients in Japan (13).

Of the vectorborne rickettsial diseases in China, human scrub typhus and murine typhus frequently occur in Zhejiang Province, and spotted fever group rickettsiosis probably occurs but has gone relatively unnoticed. Because the clinical symptoms of spotted fever and scrub typhus are similar, some SFGR infections have likely been diagnosed as scrub typhus. We found that the blood specimens of $7 \mathrm{fe}-$ brile patients were negative for the targeted PCR fragments but showed a $>4$-fold increase in antibody titer to SFGR.
Although these results suggest SFGR infection, we cannot conclude these 7 patients were infected with $R$. japonica.

In summary, $R$. japonica infections occur in Zhejiang Province, China. These infections are likely more broadly distributed throughout the mainland areas than had been previously realized. Improvements in JSF clinical diagnosis and human epidemiologic surveillance are urgently needed in China.

\section{Acknowledgments}

We thank our colleagues in the Linan First People's Hospital, Zhejiang Province, Linan, China, for their assistance in specimen collection.

\section{About the Author}

Dr. Lu is a principal investigator at the Zhejiang Province Center for Disease Control and Prevention, Hangzhou, China. Her research interests include microbiology, epidemiology, and the ecology of tickborne diseases.

\section{References}

1. Uchida T, Uchiyama T, Kumano K, Walker DH. Rickettsia japonica sp. nov., the etiological agent of spotted fever group rickettsiosis in Japan. Int J Syst Bacteriol. 1992;42:303-5. http://dx.doi.org/10.1099/00207713-42-2-303

2. Mahara F. Three Weil-Felix reaction OX2 positive cases with skin eruptions and high fever [in Japanese]. J Anan Med Assoc. 1984;68:4-7.

3. Camer GA, Alejandria M, Amor M, Satoh H, Muramatsu Y, Ueno H, et al. Detection of antibodies against spotted fever group Rickettsia (SFGR), typhus group Rickettsia (TGR), and Coxiella burnetii in human febrile patients in the Philippines. Jpn J Infect Dis. 2003;56:26-8. PubMed

4. Chung MH, Lee SH, Kim MJ, Lee JH, Kim ES, Lee JS, et al. Japanese spotted fever, South Korea. Emerg Infect Dis. 2006;12:1122-4. http://dx.doi.org/10.3201/eid1207.051372 
5. Gaywee J, Sunyakumthorn P, Rodkvamtook W, Ruang-areerate T, Mason CJ, Sirisopana N. Human infection with Rickettsia sp. related to $R$. japonica, Thailand. Emerg Infect Dis. 2007;13:657-9. http://dx.doi.org/10.3201/eid1304.060585

6. Fang LQ, Liu K, Li XL, Liang S, Yang Y, Yao HW, et al. Emerging tick-borne infections in mainland China: an increasing public health threat. Lancet Infect Dis. 2015;15:1467-79. http://dx.doi.org/10.1016/S1473-3099(15)00177-2

7. Dong X, El Karkouri K, Robert C, Raoult D, Fournier PE. Genomic analysis of Rickettsia japonica strain YHT. J Bacteriol. 2012;194:6992. http://dx.doi.org/10.1128/JB.01928-12

8. Matsutani M, Ogawa M, Takaoka N, Hanaoka N, Toh H, Yamashita A, et al. Complete genomic DNA sequence of the East Asian spotted fever disease agent Rickettsia japonica. PLoS One. 2013;8:e71861. http://dx.doi.org/10.1371/journal.pone.0071861

9. Sun J, Lin J, Gong Z, Chang Y, Ye X, Gu S, et al. Detection of spotted fever group rickettsiae in ticks from Zhejiang Province, China. Exp Appl Acarol. 2015;65:403-11. http://dx.doi.org/ 10.1007/s10493-015-9880-9
10. Meng Z, Jiang LP, Lu QY, Cheng SY, Ye JL, Zhan L. Detection of co-infection with Lyme spirochetes and spotted fever group rickettsiae in a group of Haemaphysalis longicornis [in Chinese]. Zhonghua Liu Xing Bing Xue Za Zhi. 2008;29:1217-20.

11. Cheng SY, Zheng SQ, Meng Z, Ye XD, Jiang LP, Wang ZQ, et al. Analysis of DNA sequences of spotted fever group Rickettsia detected from ticks in Zhejiang Province [in Chinese]. Dis Surveil. 2010;25:466-8.

12. Li J, Hu W, Wu T, Li HB, Hu W, Sun Y, et al. Japanese spotted fever endemic in East China, 2013. Emerg Infect Dis. 2018 Nov [cited 2018 Aug 27]. https://doi.org/10.3201/ eid2411.170264

13. Mahara F. Japanese spotted fever: report of 31 cases and review of the literature. Emerg Infect Dis. 1997;3:105-11. http://dx.doi.org/ 10.3201/eid0302.970203

Address for correspondence: Qunying Lu, Zhejiang Province Center for Disease Control and Prevention, No. 3399 Binsheng Rd, Hangzhou, 310052 China; email: lqycdc@163.com

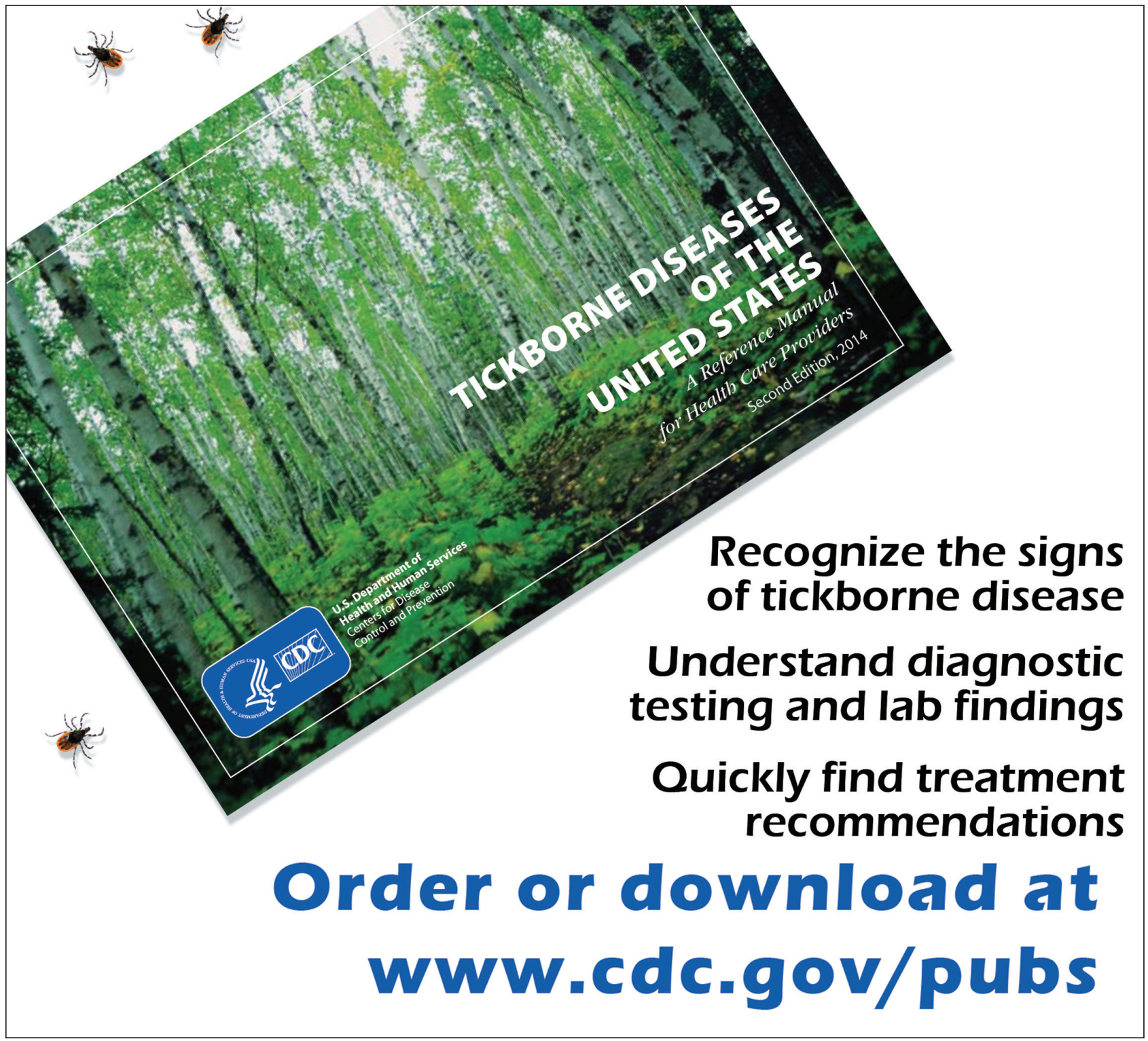

